\title{
Las TIC en la educación superior durante la pandemia de la COVID-19.
}

\section{Use of ICT in Higher Education during the COVID-19 Pandemic.}

Las TIC en la educación superior.

Stephanie Judith Gellibert Merchán ${ }^{(1)}$

Selena Esther Zapata Mora ${ }^{(2)}$

Janeth Pilar Díaz Vera ${ }^{(3)}$

(1) Universidad de Guayaquil. Ecuador. e-mail: stephanie.gellibertm@ug.edu.ec

https://orcid.org/0000-0003-0963-5531)

(2) Universidad de Guayaquil. Ecuador. e-mail: selena.zapatam@ug.edu.ec

https://orcid.org/0000-0002-0829-642X)

(3) Universidad de Guayaquil. Ecuador. e-mail:janeth.diazv@ug.edu.ec

https://orcid.org/0000-0001-8750-0216).

Contacto:stephanie.gellibertm@ug.edu.ec

\section{Resumen}

Las Tecnologías de la Información y Comunicación a lo largo de los últimos años han tomado gran importancia en el ámbito educativo puesto que cuenta con una gran gama de aplicaciones y herramientas que se pueden usar para hacer dinámica la educación. La presente investigación tiene por objetivo analizar el impacto del uso de las TIC en la modalidad de clases en línea de la Universidad de Guayaquil en tiempo de pandemia de la COVID-19 en Ecuador. Por causa de la pandemia la universidad tuvo que realizar un cambio apresurado de la modalidad presencial por una modalidad en línea para evitar el contagio en la comunidad universitaria pero esto implico nuevos retos tanto para estudiantes como docentes que no contaban con los conocimientos o recursos tecnológicos necesarios para realizar esta transición. Se realizó un estudio exploratorio y correlacional, donde se incluyeron 246 docentes y 276 estudiantes de las diferentes facultades de la Universidad de Guayaquil a los cuales se les aplicó una encuesta en línea donde se integraron 
diferentes tipos de preguntas en un cuestionario para de esa forma recolectar información necesaria y posteriormente realizar el análisis de la investigación. Los principales resultados destacanque las TIC son de gran aporte para el proceso de enseñanza-aprendizaje en la educación superior, pero para hacer un correcto uso de las tecnologías se debe adquirir competencias digitales.

Palabras clave: Análisis, TIC, clases línea, competencias digitales.

\begin{abstract}
The information and communication technologies over the last years have taken great importance in the educational field since it has a wide range of applications and tools that can be used to make education dynamic. The present research aims to make an analysis of the impact on the use of ICT in the modality of online classes at the University of Guayaquil in time of the COVID-19 pandemic in Ecuador. Due to the pandemic the university had to make a hasty change from the face-to-face modality to an online modality to avoid contagion in the university community but this implied new challenges for both students and teachers who did not have the knowledge or technological resources needed to make this transition. An exploratory and correlational study is presented below, it included 246 teachers and 276 students from the different faculties of the University of Guayaquil. An online survey was applied to these students and different types of questions were integrated into a questionnaire to collect the necessary information and later analyze the research. As a result, ICT was obtained as a major contribution to the teaching-learning process in higher education, but digital skills must be acquired to make proper use of ICTs.
\end{abstract}

Key Words: Analysis, ICT, online classes, digital competences.

\title{
Introducción
}

A finales del año 2019 por causa de un repentino brote de un enemigo imperceptible a la vista nombrado COVID-19,el cual tuvo origenen la ciudad de Wuhan, China, y se ha propagado en la mayoría de los países a nivel global en un corto lapso de tiempo; lo cual es una amenaza para la salud. Los gobernantes de cada país implementaron medidas emergentes y continúan realizando acciones contra esta pandemia, con el proposito de proteger las vidas de su población (Abreu, 2020).

Cabe señalar que las actividades docentes se han visto afectados por la pandemia de la COVID-19, con la suspensión de las clases presenciales, aplazando el inicio del siguiente periodo lectivo. Por este motivo, las unidades educativas recurrieron a la experimentación de nuevas estrategias 
enfocadas a la educación virtual y así poder continuar con el proceso de enseñanza-aprendizaje (Picardo, 2020).

En Ecuador, la Universidad de Guayaquil (UG), cuenta con más de 67000 estudiantes registrados; su medida de cambio fue la transición de la modalidad presencial a virtual implementando las tecnologías de la información y comunicación (TIC), entre estas se puede hacer mención a las plataformas digitales como, una herramienta de videoconferencia y audioconferencia para que los profesores y alumnos puedan realizar las clases virtuales de manera interactiva; en la alta casa de estudio utilizan la plataforma Moodle.

En este sentido el Vicerrectorado de Formación Académica y Profesional (VIFAP) de la Universidad de Guayaquil, elaboro una Guía Metodológica Académica para la educación en línea UG 2020", conformada por directrices, lineamientos y conceptos fundamentales, dirigido a todos los docentes de la institución, y la implementación del proceso de enseñanza-aprendizaje en modalidad en línea haciendo uso adecuado de las herramientas tecnológicas que dispone la institución (Universidad de Guayaquil, 2020).

El presente artículo tiene por objetivo analizar el impacto del uso de las TIC en la modalidad de clases en línea de la Universidad de Guayaquil en tiempo de pandemia de la COVID-19 en Ecuador.

\section{Universidad de Guayaquil y su transición de lo presencial o lo virtual}

La Universidad de Guayaquil pese a las exigencias académicas requeridas durante varios años no había realizado un cambio sumamente importante respecto a la aplicación de las TIC en la docencika, en el 2020 a causa de la pandemia global y al decreto de estado de excepciónimpuesto por el gobierno, vieron la necesidad de implementar cambios para acelerar la transición de la modalidad presencial a la virtual.

En este sentido la universidad ha realizado acciones de capacitación a los docentes de las diferentes facultades sobre metodologías, diseño curricular y de investigación; pero tanto a los docentes como estudiantes no se le ha impartido cursos o capacitaciones con respecto al desarrollo de competencia digitales, para que puedan adquirir las habilidades y destrezas en el uso de recursos tecnológicos que les permita fortalecer la docencia y el aprendizaje. 


\section{Las Tecnologías de la Información y Comunicación en la EducaciónSuperior}

Las TIC son herramientas valiosas en la docencia, la mediación tecnológica se lleva a cabo en plataformas virtuales, páginas web institucionales, sistemas de administración de aprendizaje (LMS por sus siglas en inglés: Learning Management System) a través de los foros virtuales, correo electrónico, trabajo colaborativo en herramientas en línea, videoconferencias, entre otros (Contreras, González, \& Fuentes, 2011;Contreras, 2017).

En este sentido, García et al.,(2018), asevera que las TIC fortalecen la comunicación y la interacción con actividades didácticas que propician una mayor motivación en los educandos, una de estas herramientas son las redes sociales, las plataformas digitales para las videoconferencias, las cuales constituyen un servicio que permite poner en contacto a un grupo de personas mediante sesiones interactivas para que puedan ver y escuchar las intervenciones de los docentes.

\section{Material y métodos}

El trabajo investigativo se ejecutó bajo el análisis exploratorio, que permitió realizar la formulación del problema tomando como base la situación que generó la pandemia de la COVID-19 en la Universidad de Guayaquil. El estudio siguió un diseño correlacional, que cual determinar la relación existente entre las variables y dimensiones del problema, objetivos, premisa y contexto de la investigación a través de los datos obtenidos en el levantamiento de la información.

La investigación se desarrolló bajo un enfoque mixto cualitativo-cuantitativo, se tomó como referencia la técnica de levantamiento de información conocida como encuesta, a fin de contemplar la obtención de datos estadísticos de la muestra evaluada, establecer criterios de comprensión para brindar respuesta al problema y cumplir con los objetivos planteados.

La población en este estudio, se tomo como eje principal, la cantidad total de docentes y estudiantes que posee la Universidad de Guayaquil en sus 17 facultades en el periodo académico 2020-2021 CI. A continuación, se detalla la población de la investigación en la tabla 1 corresponde a los docentes y la tabla 2 los estudiantes:

\begin{tabular}{|l|l|c|}
\hline no. & Facultad & Cant. \\
\hline 1 & Arquitectura Y Urbanismo & 79 \\
\hline 2 & Ciencias Administrativas & 335 \\
\hline 3 & Ciencias Agrarias & 27 \\
\hline 4 & Ciencias Económicas & 78 \\
\hline
\end{tabular}




\begin{tabular}{|l|l|c|}
\hline 5 & Ciencias Matemáticas y Físicas & 160 \\
\hline 6 & Ciencias Medicas & 470 \\
\hline 7 & Ciencias Naturales & 47 \\
\hline 8 & Ciencias Psicológicas & 48 \\
\hline 9 & Ciencias Químicas & 41 \\
\hline 10 & Comunicación Social & 114 \\
\hline 11 & Educación Física Deporte y Recreación & 39 \\
\hline 12 & Filosofía, Letras y Ciencias de la Educación & 245 \\
\hline 13 & Ingeniería Industrial & 116 \\
\hline 14 & Ingeniería Química & 92 \\
\hline 15 & Jurisprudencia Ciencias Sociales y Políticas & 118 \\
\hline 16 & Medicina Veterinaria Y Zootecnia & 21 \\
\hline 17 & Odontología & 103 \\
\hline & $\begin{array}{l}\text { Arquitectura Y Urbanismo | Ciencias } \\
\text { Económicas }\end{array}$ \\
\hline & Arquitectura Y Urbanismo | Odontología & 1 \\
\hline & Ciencias Agrarias | Comunicación Social & 1 \\
\hline & Ciencias Médicas | Comunicación Social & 1 \\
\hline & Ciencias Químicas | Ingeniería Industrial & 1 \\
\hline Total & & 2138 \\
\hline
\end{tabular}

Tabla 1. Población de la Investigación - Docentes

\begin{tabular}{|l|l|c|}
\hline No. & Facultad & Cant. \\
\hline 1 & Arquitectura y Urbanismo & 1720 \\
\hline 2 & Ciencias Administrativas & 10393 \\
\hline 3 & Ciencias Agrarias & 783 \\
\hline 4 & Ciencias Económicas & 2322 \\
\hline 5 & Ciencias Matemáticas y Físicas & 5437 \\
\hline 6 & Ciencias Medicas & 8302 \\
\hline 7 & Ciencias Naturales & 959 \\
\hline 8 & Ciencias Psicológicas & 2007 \\
\hline 9 & Ciencias Químicas & 1417 \\
\hline 10 & Comunicación Social & 3332 \\
\hline 11 & Educación Física Deporte y Recreación & 1190 \\
\hline 12 & Filosofía, Letras y Ciencias De La & 6658 \\
\hline 13 & Educación & 4157 \\
\hline 14 & Ingeniería Industrial & 2765 \\
\hline 15 & Jurisprudeniería Química & 3664 \\
\hline 16 & Medicina Veterinaria y Zootecnia & 560 \\
\hline 17 & Odontología & 1897 \\
\hline & Arquitectura y Urbanismo | Ciencias & 1 \\
& Matemáticas y Físicas & 1 \\
\hline & Ciencias Médicas | Ciencias Psicológicas & 1 \\
\hline & $\begin{array}{l}\text { Ciencias Psicológicas | Comunicación } \\
\text { Social }\end{array}$ & \\
\hline & Comunicación Social | Jurisprudencia & 1 \\
\hline
\end{tabular}




\begin{tabular}{|l|l|}
\hline Ciencias Sociales y Políticas & \\
\hline TOTAL & 57567 \\
\hline
\end{tabular}

Tabla 2. Población de la Investigación - Estudiantes en la Universidad de Guayaquil en el periodo académico 2020-2021.

Para obtener una muestra del total de estudiantes y docentes de la Universidad de Guayaquil se utilizó la siguiente fórmula:

$$
n=\frac{\mathbf{m}}{e^{2}(\boldsymbol{m}-\mathbf{1})+\mathbf{1}}
$$

Donde:

m: Tamaño de la población

e: error de estimación

n: Tamaño de la muestra

Para poder calcular la muestra de la investigación se ha considerado dividirla en dos estratos, que son estudiantes y docentes de la Universidad de Guayaquil.

\section{Muestra de Docentes}

Datos

$$
\begin{gathered}
m_{1}: 2138 \\
e: 6 \% \\
n_{1}=\frac{m_{1}}{e^{2}\left(m_{1}-1\right)+1} \\
n_{1}=\frac{2138}{0.06^{2}(2138-1)+1} \\
n_{1}=\frac{2138}{0.0036(2137)+1} \\
n_{1}=\frac{2138}{7.6932+1}
\end{gathered}
$$




$$
\begin{aligned}
& n_{1}=\frac{2138}{8.6932} \\
& n_{1}=245.93
\end{aligned}
$$

\section{Muestra de Estudiantes}

Datos

$m_{2}: 57567$

$e: 6 \%$

$$
\begin{gathered}
n_{2}=\frac{m_{2}}{0.06^{2}\left(m_{2}-1\right)+1} \\
n_{2}=\frac{57567}{0.06^{2}(57567-1)+1} \\
n_{2}=\frac{57567}{0.0036(57566)+1} \\
n_{2}=\frac{57567}{207.237+1} \\
n_{2}=\frac{57567}{208.237} \\
n_{2}=276.45
\end{gathered}
$$

La técnica utilizada para la recolección de datos del proyecto de investigación es la encuesta, la cual cuenta con un total de 10 preguntas dirigidas a los docentes y 10 preguntas dirigidas a los estudiantes. Para poder generar la encuesta en línea se hizo uso de la herramienta Google Forms, la cual permite compartir el link de la encuesta a través de medios electrónicos como el correo institucional y redes sociales con los 246 docentes y 276 estudiantes.

\section{Resultados}

Los participantes en el estudio fueron 246 docentes y 276 estudiantes de la Facultad de las diferentes facultades de la UG, con el propósito de analizar si existe un impacto del uso de las TIC 
en la modalidad de clases en línea de la Universidad de Guayaquil en tiempo de pandemia de la COVID-19.

Al realizar el análisis de la pregunta relacionado con el nivel de de apropiación de las TIC que usted tiene, se puede visualizar que el $48 \%$ de los docentes consideran tener un nivel intermedio de apropiación de las TIC y el 25\% consideran tener un nivel Alto. Los resultados reflejan que el 73\% de los docentes de la UG cuentan con un nivel intermedio-alto sobre el uso de las TIC, lo que indica que la mayoría tiene conocimiento medio sobre cómo manejar las diferentes aplicaciones de una manera correcta, figura 1.

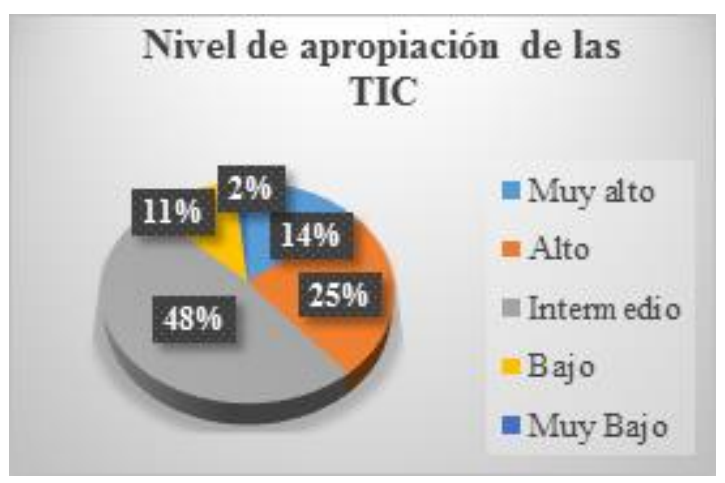

Figura 1. Nivel de apropiación de las TIC en docentes

En cuanto a si han recibido capacitación sobre el tema de la competencias por parte de la Universidad de Guayaquil antes de la pandemia de la COVID-19, se encontró que el 67\% de docentes ha recibido capacitaciones acerca del tema de las competencias digitales por parte de la Universidad de Guayaquil antes del cambio de modalidad lo cual indicaría que la mayoría de los docentes deberían tener conocimiento sobre el correcto uso de las TIC al momento de impartir las clases virtuales.

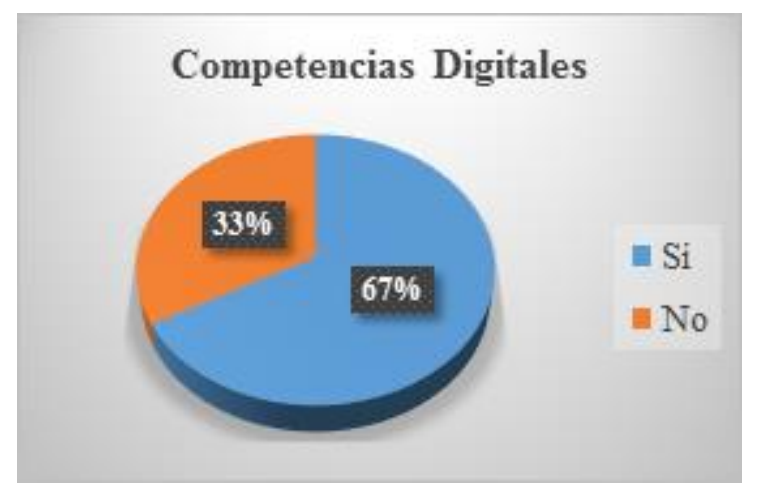

Figura 2. Capacitación del profesorado en lo relativo a las competencias digitales 
En lo concerniente al nivel de interacción entre docente-estudiante en las clases virtuales vs clases presenciales, se obtuvo como resultados que el 33,74\% de los docentes consideran que el nivel de interacción se encuentra en un punto intermedio, y el 23,98\% consideran que el nivel de interacción con los estudiantes es bajo. Lo cual indica que no existe una buena interacción durante las clases virtuales entre los involucrados, lo que afecta directamente el proceso de enseñanza-aprendizaje, tabla 3 .

\begin{tabular}{|l|l|l|}
\hline Opción & $\begin{array}{l}\text { Docentes } \\
\text { Encuestados }\end{array}$ & Porcentaje \\
\hline 1 & 52 & $21,14 \%$ \\
\hline 2 & 59 & $23,98 \%$ \\
\hline 3 & 83 & $33,74 \%$ \\
\hline 4 & 34 & $13,82 \%$ \\
\hline 5 & 18 & $7,32 \%$ \\
\hline Total & 246 & $100 \%$ \\
\hline
\end{tabular}

Tabla 3. Nivel de interacción entre docente-estudiante

La reflexión en torno a la calidad de la educación actualmente en la universidad de Guayaquil, es importante destacar a que no solo se hace referencia a las clases virtuales sino a la calidad de la educación. Lo que se observa que el $48 \%$ de estudiantes opinan que la calidad de educación en la UG es regular, mientras que el $37 \%$ opinan que la calidad de la educación en la UG es buena. A manera de reglexión, casi la mitad de los estudiantes opinan que la calidad de educación en el centro, es considerada por los estudiantes como regular, figura 3.

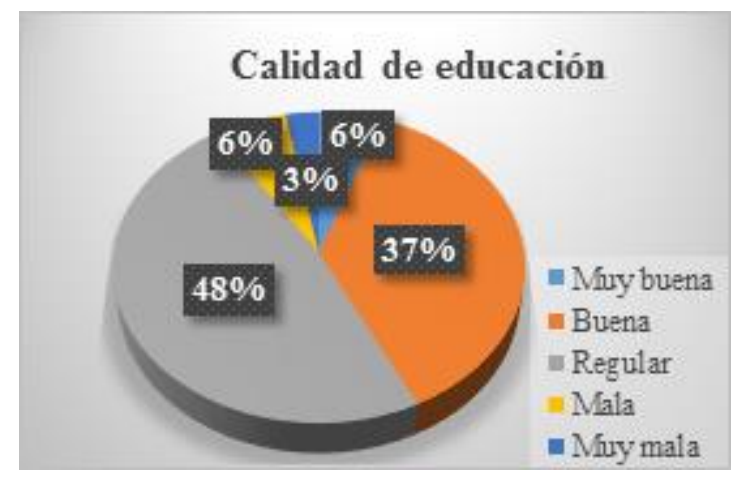

Figura 3. Calidad de educación en la UG

En lo concerniente a lapregunta relacionada con la capacitación en tornoa las competencias digitales, la cuales proveen de habilidades para hacer un uso de las tecnología. Los resultados revelan en la tabla 4 , que el $87,3 \%$ de estudiantes nunca han recibido capacitaciones acerca de las Competencias Digitales por parte de la Universidad de Guayaquil lo cual indicaría que la mayoría 
de los estudiantes no cuentan con el conocimiento sobre las competencias digitales y sobre cómo hacer un correcto uso de las TIC en las clases virtuales. Cabe destacar que solo un 12,7\%, señala haber recibido capacitación.

\begin{tabular}{|l|l|l|}
\hline Opción & $\begin{array}{l}\text { Estudiantes } \\
\text { Encuestados }\end{array}$ & Porcentaje \\
\hline Sí & 35 & $12,7 \%$ \\
\hline No & 241 & $87,3 \%$ \\
\hline Total & 276 & $100 \%$ \\
\hline
\end{tabular}

Tabla 4. Capacitaciones competencia digitales en estudiantes

De acuerdo con el análisis de la figura4, acerca de que si consideran que existe el mismo nivel de aprendizaje en la modalidad en línea está al mismo nivel de la educación presencial, se observa que el $38,0 \%$ de los estudiantes consideran que el nivel de aprendizaje se encuentra en un punto intermedio, y el 26,0\% opinan que están en desacuerdo con respecto a que el nivel de aprendizaje de las clases virtuales está al mismo nivel que las clases presenciales. Lo cual indica que los estudiantes consideran que el nivel de aprendizaje se encuentra en un punto intermedio-bajo con respecto a las clases presenciales.

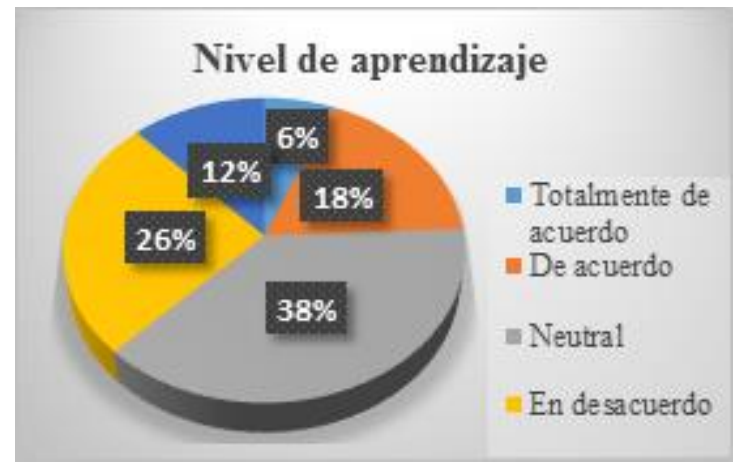

Figura 4. Nivel de aprendizaje modalidad en línea vs presencial

El análisis de la pregunta, sobre qué tipo de modalidad de estudio prefieren, dio como resultado que $78 \%$ de los estudiantes de la Universidad de Guayaquil prefieren la educación presencial, a pesar de contar con los recursos tecnológicos para acceder a las clases virtuales, figura 5. 


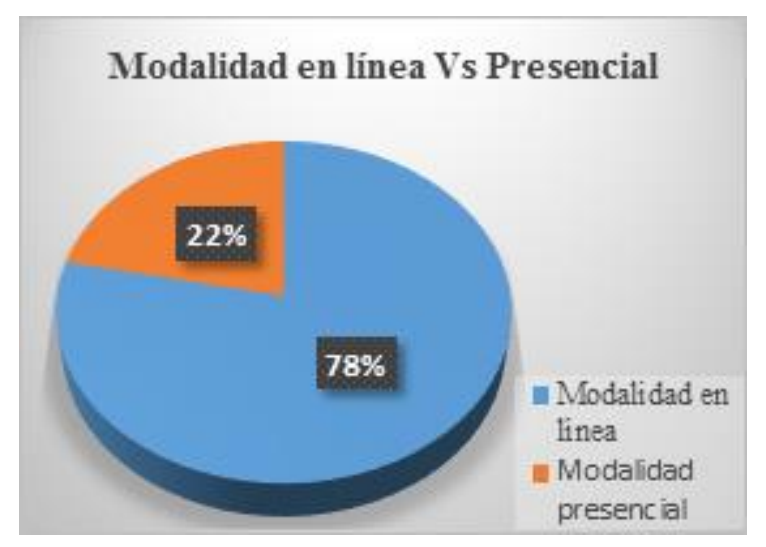

Figura 5. Tipo modalidad que prefieren los estudiantes

\section{Discusión}

La reflexión en tormo al nivel de apropiación de las tecnologías de los docentes, se puede plantear que los profesores de las diferentes facultades de la Universidad de Guayaquil según su criterio cuentan con un nivel intermedio a alto de apropiación de las TIC, a pesar de que cierto porcentaje de docentes no fueron capacitados sobre las competencias digitales antes de la pandemia de la COVID-19, además, los docentes consideran que existe un nivel intermedio a bajo de interacción entre docentes-estudiantes.

Se concuerda con otros estudios que hacen alusión a las herramientas tecnológicas y su incorpracióna la actividad docente, y cómo la percepción que los docentes tienen del rol de las TIC y de su importancia en las actividades académicas inciden en la apropiación de dichas herramientas, también hacen referencia al uso de WhatsApp para fortalecer la comunicación y la docencia en línea. También refieren que a través de estas herramientas digitales, se realizan evaluaciones, talleres, trabajos en línea, foros de discusión y videoconferencias (Tapasco\& Giraldo 2017).

En esta experiencia se coincide con otras investigaciones realizadas por Pozo \& Tejada (2018), en lo relativo a las competencias difitales del profesorado, los resultados indican un dominio mediobajo en las competencias digitales que corresponden con el rol de docencia (planificación, el desarrollo y conducción de experiencias de aprendizaje y la evaluación con apoyo de las TIC).

Por otra parte, los estudiantes de las diferentes facultades de la Universidad de Guayaquil consideran que la calidad de la educación de la institución es regular a buena, también manifestaron en su mayoría que no han recibido capacitaciones sobre las competencias digitales por parte de la universidad, además, los estudiantes estiman que el nivel de aprendizaje en la modalidad en línea se 
encuentra en un rango entre intermedio a bajo con respecto a la educación presencial, y por último la mayoría de estudiantes prefieren la modalidad presencial.

Estos resultados concuerdan con otras investigaciones que reflejan que el grado de percepción de los estudiantes con respecto a la modalidad presencial y semipresencial es alto, lo que permite extender y mejorar la experiencia (Aguilar, Fuentes, Justo, \& Rivera 2019).

\section{Conclusiones}

La investigación realizada genera varias conclusiones, se pudo deterimar que las herramientas tecnológicas son viables para aplicarlas en la enseñanza superior; para esto se debe adquirir competencias digitales o tener un nivel de apropiación de las TIC intermedio para usarlas de forma eficiente.

El estudio desarrollado permitió mostrar que los docentes y estudiantes de la Universidad de Guayaquil, no se encontraban preparados para el cambio repentino de modalidad de clases, pese a que los docentes y estudiantes tienen conocimientos de las TIC; y poseen los recursos tecnológicos, eso no fue suficiente para lograr que el proceso de enseñanza-aprendizaje fuera óptimo debido a que no existió una buena planificación, existen ciertas dificultades en el uso de las plataformas lo que ocasiona que no se dé una buena interacción durante las clases sincrónicas entre los docentes y estudiantes.

Tomando en cuenta los puntos más relevantes de la investigación se puede determinar que las TIC tienen un gran impacto en la modalidad en línea debido a que se visualizó un incremento sustancial en su implementación en las clases virtuales frente al tiempo pre-cuarentena donde su uso era limitado durante el proceso de enseñanza-aprendizaje.

\section{Bibliografía}

Abreu, J. L. (2020, 15 mayo). Tiempos deCoronavirus: La Educación en Línea como Respuesta a la Crisis. Daena: International Journal of Good Conscience. Recuperado de http://www.spentamexico.org/v15-n1/A1.15(1)1-15.pdf

Aguilar, W., Fuentes, M., Justo, A., \& Rivera, R. (2019). Percepción de los Estudiantes acerca de la Modalidad Semipresencial en la Enseñanza de las Ciencias Básicas de la Ingeniería. Un Estudio de Caso Universitario. Formación universitaria, 12(3), 15-

26. https://dx.doi.org/10.4067/S0718-50062019000300015 
Picardo, O. (2020, mayo). COVID-19: Una mirada interdisciplinaria a la pandemia. REDICCES. Recuperado de http://www.redicces.org.sv/jspui/handle/10972/4149

Universidad de Guayaquil. (2020a, mayo). Memorando Nro. UG-CFAP-2020-0980-M.

Contreras, L. , González, K., \& Fuentes, H. (2011). Uso de las TIC y especialmente del blendedlearning en la enseñanza universitaria. Educación y desarrollo social, 151-160. https://doi.org/10.18359/reds.898

Contreras, J. L. R. (2017). Importancia de las TIC en enseñanza de las matemáticas. Revista Matemática de la Universidad del Atlántico, 4(2), 1. Recuperado de: http://investigaciones.uniatlantico.edu.co/revistas/index.php/MATUA/article/view/1861

García, M., Reyes, J., \& Godínez, G. (2018). Las TIC en la educación superior, innovaciones y retos / The ICT in higher education, innovations and challenges.RICSH Revista Iberoamericana de las Ciencias Sociales y Humanísticas, 6(12), 17. https://doi.org/10.23913/ricsh.v6i12.135

Pozos, K. \& Tejada ez, J. (2018). Competencias digitales docentes en educación superior: niveles de dominio y necesidades formativas. Revista Digital de Investigación en Docencia Universitaria, 12(2), 59-87. doi: http://dx.doi.org/10.19083/ridu.2018.712

Levano, L., Sanchez, S., Guillén, P., Tello, S., Herrera, N., \&Collantes, Z. (2019). Competencias digitales y educación. La Scientific Electronic Library Online, 7(2). Recuperado de: http://www.scielo.org.pe/scielo.php?pid=S2307$79992019000200022 \&$ script $=$ sci_abstract\&tlng=en

Mosquera, I. (2018). El necesario desarrollo de la competencia digital del profesorado. Fundación Universidad Internacional de Rioja - UNIR Revista. Recuperado de: https://www.unir.net/educacion/revista/noticias/el-necesario-desarrollo-de-la-competenciadigital-del-profesorado/549203517804/

Smith, S. (2020). Diferentes tipos de clases en línea. Understood.Recuperado de: https://www.understood.org/es-mx/school-learning/choosing-starting-school/homeschooling/different-types-of-online-classrooms 
Tapasco, O., \& Giraldo, J. (2017). Estudio Comparativo sobre Percepción y uso de las TIC entre Profesores de Universidades Públicas y Privadas. Revista Formación Universitaria, 10(2), 0312. Recuperado de: https://dx.doi.org/10.4067/S0718-50062017000200002

Valencia, T., Serna, A., Ochoa, S., Caicedo, A., Montes, J., \& Chávez, J. (2016). Competencias y estándares TIC desde la dimensión pedagógica: una perspectiva desde los niveles de apropiación de las TIC en la práctica educativa docente. Recuperado de: https://unesdoc.unesco.org/ark:/48223/pf0000259508? posInSet=1\&queryId=8718de75-4cf74898-b35a-48b5b9b11b79 